\title{
Serotype distribution of Streptococcus mutans a pathogen of dental caries in cardiovascular specimens from Japanese patients
}

Correspondence

Takashi Ooshima

ooshima@dent.osaka-u.ac.jp

Received 6 November 2006

Accepted 19 December 2006

\author{
Kazuhiko Nakano, ${ }^{1}$ Hirotoshi Nemoto, ${ }^{1}$ Ryota Nomura, ${ }^{1}$ Hiromi Homma, ${ }^{1}$ \\ Hideo Yoshioka, ${ }^{2}$ Yasuhiro Shudo, ${ }^{3}$ Hiroki Hata, ${ }^{3}$ Koichi Toda, ${ }^{3}$ \\ Kazuhiro Taniguchi, ${ }^{3}$ Atsuo Amano ${ }^{4}$ and Takashi Ooshima ${ }^{1}$ \\ ${ }^{1}$ Department of Pediatric Dentistry, Osaka University Graduate School of Dentistry, \\ 1-8 Yamada-oka, Suita, Osaka 565-0871, Japan \\ ${ }^{2}$ Department of Dentistry and Oral Surgery, Osaka Rosai Hospital, 1179-3 Nagasane-cho, Sakai, \\ Osaka 591-0825, Japan \\ ${ }^{3}$ Department of Cardiovascular Surgery, Osaka Rosai Hospital, 1179-3 Nagasane-cho, Sakai, \\ Osaka 591-0825, Japan \\ ${ }^{4}$ Department of Oral Frontier Biology, Osaka University Graduate School of Dentistry, \\ 1-8 Yamada-oka, Suita, Osaka 565-0871, Japan
}

The involvement of oral bacteria in the pathogenesis of cardiovascular disease has been studied, with Streptococcus mutans, a pathogen of dental caries, detected in cardiovascular lesions at a high frequency. However, no information is available regarding the properties of $S$. mutans detected in those lesions. Heart valve specimens were collected from 52 patients and atheromatous plaque specimens from 50 patients, all of whom underwent cardiovascular operations, and dental plaque specimens were taken from 41 of those subjects prior to surgery. Furthermore, saliva samples were taken from 73 sets of healthy mothers $(n=73)$ and their healthy children $(n=78)$. Bacterial DNA was extracted from all specimens, then analysed by PCR with S. mutans-specific and serotype-specific primer sets. The detection rates of $S$. mutans in the heart valve and atheromatous plaque specimens were 63 and $64 \%$, respectively. Non-c serotypes were identified with a significantly higher frequency in both cardiovascular and dental plaque samples from the subjects who underwent surgery as compared to serotype $c$, which was detected in $70-75 \%$ of the samples from the healthy subjects. The serotype distribution in cardiovascular patients was significantly different from that in healthy subjects, suggesting that S. mutans serotype may be related to cardiovascular disease.

\section{INTRODUCTION}

Streptococcus mutans, a major pathogen of dental caries, has been detected in heart valves and isolated from the blood of patients with infective endocarditis (IE), suggesting its close relationship with IE (Vose et al., 1987; Ullman et al., 1988; Gauduchon et al., 2001; Nomura et al., 2006). $S$. mutans is classified into four serotypes $(c / e / f / k)$ based on the chemical composition of its cell surface serotypespecific rhamnose-glucose polymers (RGPs), which form a backbone of rhamnose polymers with side chains of glucose polymers (Hamada \& Slade, 1980; Nakano et al., 2004a). Serotype $c$ is reported to be the most prevalent in oral isolates at approximately 70-80 \%, followed by $e$, $f$ and k (Hamada et al., 1980; Hirasawa \& Takada, 2003; Shibata

Abbreviations: IE, infective endocarditis; RGP, rhamnose-glucose polymer. et al., 2003; Nakano et al., 2004b). In our previous study, four $S$. mutans strains isolated from the blood of patients with bacteraemia after tooth extraction or IE were characterized; however, none of those strains were classified as serotype $c$ (Fujiwara et al., 2001).

The serotype-specific RGPs of S. mutans were demonstrated to play important roles in streptococcal adherence to human monocytic and fibroblastic cells, and speculated to be its most efficient cytokine-stimulating components (Engels-Deutsch et al., 2003). In addition, the hydrophilic nature of RGPs is known to be associated with the resistance to phagocytosis by human polymorphonuclear leukocytes (Tsuda et al., 2000). Further, RGPs were shown to possess properties allowing them to bind directly to human platelets and trigger their aggregation in a dose-dependent manner (Chia et al., 2004). In our previous study, serotype $k$ strains featured a drastic reduction in the amount of 
glucose side chains and were shown to be less susceptible to phagocytosis by human polymorphonuclear leukocytes than the parent strain (Nakano et al., 2004a). However, the virulence of each serotype of S. mutans in blood remains to be elucidated.

Recently, the association of cardiovascular diseases and periodontitis has received attention because of results showing that periodontopathic bacterial DNA could be detected in the diseased cardiovascular tissues (Scannapieco et al., 2003). We previously reported that not only periodontopathic bacterial species, but also several streptococcal species, were detected in cardiovascular specimens, such as heart valve and atheromatous plaque, and $S$. mutans was the most frequently detected (Nakano et al., 2006a). In the present study, detection of $S$. mutans in additional specimens, as well as serotype determination of S. mutanspositive specimens, was carried out.

\section{METHODS}

Specimens from subjects undergoing a cardiovascular operation. Fifty-two heart valve and fifty atheromatous plaque samples were obtained from subjects with cardiovascular diseases who visited Osaka Rosai Hospital. Of these, 41 subjects were referred to the Department of Dentistry and Oral Surgery for a dental examination prior to cardiovascular surgery, and from whom dental plaque specimens were obtained. All of the specimens were collected from December 2004 to August 2006 according to a protocol approved by the ethics committee of Osaka Rosai Hospital. The heart valve tissue specimens were excised during a valve replacement procedure, following diagnosis of aortic regurgitation, aortic stenosis, mitral regurgitation, mitral stenosis or tricuspid regurgitation, while the atheromatous plaque specimens were collected during treatment for a thoracic or abdominal aortic aneurysm. The specimens were aseptically cut into small pieces, and bacterial DNA was extracted as described previously (Nakano et al., 2006a).

Specimens from mothers and their children. Seventy-three pairs of mothers (73 subjects) and their children (78 subjects) who came to the Pedodontics Clinic of Osaka University Dental Hospital, Osaka, Japan, from June 2005 to August 2006 were analysed. None of the children or mothers had systemic disorders. Collection of clinical specimens was carried out in accordance with a protocol approved by the ethics committee of Osaka University Dental Hospital. Whole saliva samples were taken and bacterial DNA was extracted according to previously described methods (Hoshino et al., 2004).

S. mutans detection and serotype determination. S. mutans detection was carried out using primers targeting the $g t f D$ gene (Hoshino et al., 2004) (Table 1). The serotype of each sample was determined by serotype $c$-, $e$ - or $f$-specific sets of primers (Shibata et al., 2003), as well as a serotype $k$-specific set of primers generated in our previous study (Nakano et al., 2004b). S. mutans MT8148 (c), NN2001 $(c)$, NN2002 $(e), \mathrm{NN} 2003(f)$ and FT1 $(k)$ were used as reference strains for $S$. mutans and each serotype (Nakano et al., 2004 b). Samples with a positive reaction to the $S$. mutans-specific set of primers but not with any of the serotype-specific sets of primers were confirmed using an additional S. mutans-specific set of primers based on the $g t f B$ sequence (Oho et al., 2000). In addition, sequence determination of the amplified fragments was carried out to confirm that they contained the target nucleotide alignment. The sensitivity of all the PCR methods was evaluated using diluted DNA extracted from the reference strains. In order to confirm that $S$. mutans DNA was present in the serotype undetermined specimens, a broad-range PCR assay targeting $16 \mathrm{~S}$ rRNA and sequencing analysis was carried out, as described elsewhere (Rovery et al., 2005; Nakano et al., 2006a). Briefly, PCR products amplified by broad-range 16S rRNA primers [536f (5'-CAG CAG CCG CGG TAA TAC-3') and 1050r (5'-CAC GAG CTG ACG ACA-3')] were cloned into a pGEM-T Easy vector (Promega), after which ten clones were randomly chosen and their sequences determined. To identify the bacterial species, the sequences were compared with those available in the GenBank, EMBL and DDBJ databases using the gapped BLASTN 2.0.5 program obtained from the National Center for Biotechnology Information server (http:/www.ncbi.nlm.nih.gov/BLAST/). Identification at the species level was determined when the sequence similarity was greater than $99 \%$ of that of the GenBank prototype strain sequence.

Statistical analysis. Fisher's exact probability test was used for statistical analysis of the comparative frequency of each serotype. A $P$ value of $<0.05$ was considered significant.

Table 1. PCR primers used for $S$. mutans detection and serotype determination

\begin{tabular}{|c|c|c|c|c|}
\hline Primer & Purpose & Sequence $\left(5^{\prime}-3^{\prime}\right)$ & $\begin{array}{l}\text { Expected } \\
\text { size (bp) }\end{array}$ & Reference \\
\hline MKD-F & S. mutans detection & GGC ACC ACA ACA TTG GGA AGC TCA GTT & 433 & Hoshino et al. (2004) \\
\hline MKD-R & & GGA ATG CCG ATC AGT CAA CAG GAT & & \\
\hline$g t f B-F$ & S. mutans detection & ACT ACA CTT TCG GGT GGC TTG G & 517 & Oho et al. (2000) \\
\hline$g t f B-\mathrm{R}$ & & CAG TAT AAG CGC CAG TTT CAT C & & \\
\hline SC-F & Serotype $c$ determination & CGG AGT GCT TTT TAC AAG TGC TGG & 727 & Shibata et al. (2003) \\
\hline SC-R & & AAC CAC GGC CAG CAA ACC CTT TAT & & \\
\hline SE-F & Serotype $e$ determination & CCT GCT TTT CAA GTA CCT TTC GCC & 517 & Shibata et al. (2003) \\
\hline SE-R & & CTG CTT GCC AAG CCC TAC TAG AAA & & \\
\hline SF-F & Serotype $f$ determination & CCC ACA ATT GGC TTC AAG AGG AGA & 316 & Shibata et al. (2003) \\
\hline SF-R & & TGC GAA ACC ATA AGC ATA GCG AGG & & \\
\hline CEFK-F & Serotype $k$ determination & ATT CCC GCC GTT GGA CCA TTC C & 294 & Nakano et al. (2004b) \\
\hline K-R & & CCA ATG TGA TTC ATC CCA TAC C & & \\
\hline
\end{tabular}




\section{RESULTS AND DISCUSSION}

The minimum amounts of genomic DNA required for $S$. mutans detection and serotype determination by PCR methods used in the present study were evaluated using serial dilution of known concentrations of genomic DNA extracted from the reference strains (Fig. 1). The PCR results with $g t f D$ - and $g t f B$-based primer sets showed that the minimum amount of template DNA for $S$. mutans detection was $1-10 \mathrm{pg}$, which was also the detection limit for the template DNA for serotype clelflk.

The detection rates of $S$. mutans in heart valve and atheromatous plaque specimens were 63.4 and $64.0 \%$, respectively (Table 2). In the heart valve specimens, $60.6 \%$ of the $S$. mutans-positive samples were shown to be a single serotype, with serotype $e$ the most prevalent followed by $c, f$ and $k$. Specimens with multiple serotypes detected were also identified at a frequency of $15.2 \%$. As for the atheromatous plaque specimens, 31.2 and $59.4 \%$ of the S. mutans-positive samples were shown to be single and multiple serotypes, respectively. In addition, there were eight heart valves and three atheromatous plaque specimens that did not react to any of the serotype-specific sets of primers. Those specimens were also confirmed to have a positive reaction toward the $g t f B$-based primers. In addition, broad-range PCR and sequencing analyses showed that $S$. mutans was identified in at least one of ten clones analysed.
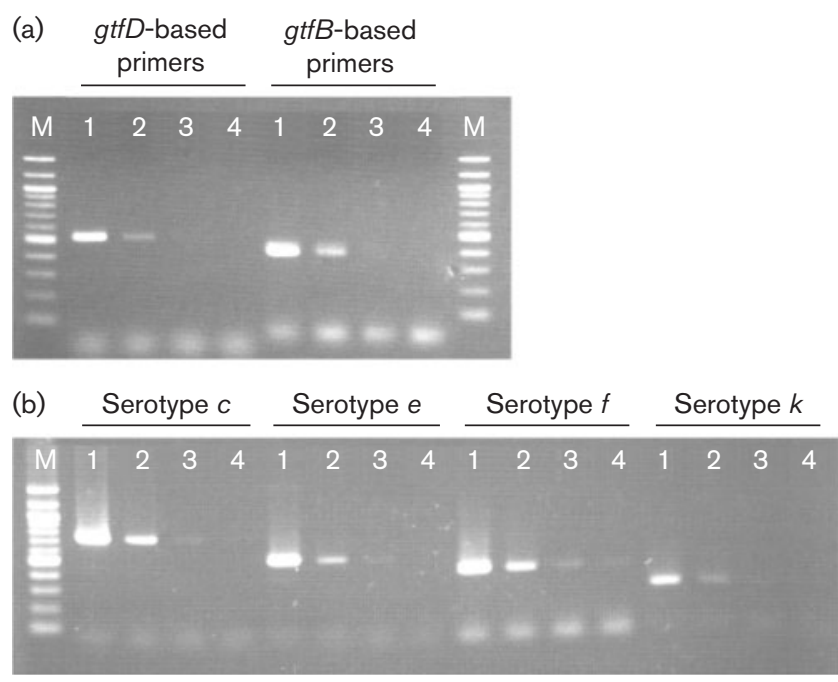

Fig. 1. Sensitivity of the PCR methods for $S$. mutans detection and serotype determination. (a) A gel showing the detection limit using the $S$. mutans-specific sets of primers. The gtfD- and gtfBbased primer set were evaluated by genomic DNA from NN2001 (serotype c). (b) A gel showing the detection limit using the serotype-specific sets of primers. The serotype $c^{-}, e^{-}, t-$, and $k-$ specific sets of primers were analysed by genomic DNA from NN2001 (c), NN2002 (e), NN2003 (f) and FT1 (k), respectively. Lanes: 1, 100 pg; 2, 10 pg; 3, 1 pg; 4, 0.1 pg; M, molecular size marker (100 bp DNA ladder).
The rates of S. mutans-positive specimens in dental plaque samples from subjects who underwent cardiovascular surgery, and in saliva samples from the mothers and their children ranged from 88.4 to $95.1 \%$ (Table 3). However, the serotype distribution in the healthy mothers and children was quite different from that in the cardiovascular surgery subjects. In the subjects who underwent surgery, the proportion of specimens containing multiple serotypes was greater than that of specimens containing a single serotype. In addition, a non- $c$ serotype was detected in cardiovascular surgery subjects more often than in the healthy children and their mothers. As for the serotype distribution in the mothers and children, specimens with a single serotype were detected more often than in the cardiovascular surgery subjects, with the detection of serotype $c$ much higher than any of the other serotypes. In addition, we were unable to classify two (5.1\%) specimens from the cardiovascular surgery subjects as any known serotype, whereas all of those from mothers and children were classified. Table 4 summarizes the serotype distribution frequency of $S$. mutans-positive specimens. The frequency of serotype $c$ in the cardiovascular specimens, as well as in the oral specimens from the subjects who underwent cardiovascular operations, was significantly lower than that of the oral specimens from the healthy mothers and children $(P<0.05)$. On the other hand, the rates for serotypes $e$ and $k$, as well as untypable specimens, among the cardiovascular specimens were significantly higher than among the oral specimens from

Table 2. Detection and serotype distribution of $S$. mutans in cardiovascular specimens

\begin{tabular}{|lcc|}
\hline & $\begin{array}{c}\text { Heart valve } \\
(\boldsymbol{n}=\mathbf{5 2})\end{array}$ & $\begin{array}{c}\text { Atheromatous } \\
\text { plaque }(\boldsymbol{n}=\mathbf{5 0})\end{array}$ \\
\hline Age (range) & $67.8 \pm 8.7(49-87)$ & $70.8 \pm 7.2(56-82)$ \\
Gender (male : & $36: 16$ & $37: 13$ \\
female) & & \\
Positive for $S$. mutans & $33 / 52(63.4 \%)^{*}$ & $32 / 50(64.0 \%)^{\star}$ \\
Single serotype & $20 / 33(60.6 \%) \dagger$ & $10 / 32(31.2 \%) \dagger$ \\
$c$ & 7 & 5 \\
$e$ & 11 & 4 \\
$f$ & 1 & 1 \\
$k$ & 1 & 0 \\
Multiple serotype & $5 / 33(15.2 \%) \dagger$ & $19 / 32(59.4 \%) \dagger$ \\
$c$ and $e$ & 3 & 10 \\
$c$ and $k$ & 0 & 3 \\
$e$ and $f$ & 0 & 1 \\
$e$ and $k$ & 2 & 3 \\
$c, e$ and $k$ & 0 & $3 / 32(9.4 \%) \dagger$ \\
Serotype undetermined & $8 / 33(24.2 \%) \dagger$ & \\
\hline
\end{tabular}

*The percentage is expressed in comparison to all (100\%) of the heart valve and atheromatous plaque specimens.

$\dagger$ The percentage is expressed in comparison to all (100\%) of the $S$. mutans-positive specimens. 
Table 3. Detection and serotype distribution of $S$. mutans in the oral cavity

\begin{tabular}{|c|c|c|c|}
\hline & $\begin{array}{l}\text { Cardiovascular } \\
\text { surgery patient } \\
\qquad(n=41)\end{array}$ & $\begin{array}{l}\text { Healthy mother } \\
\qquad(n=73)\end{array}$ & $\begin{array}{l}\text { Healthy child } \\
\qquad(n=78)\end{array}$ \\
\hline Age (range) & $66.1 \pm 7.8(49-80)$ & $37.7 \pm 4.2(28-50)$ & $7.2 \pm 2.4(2-12)$ \\
\hline Gender (male : female) & $30: 11$ & $0: 73$ & $40: 38$ \\
\hline Specimens & Dental plaque & Saliva & Saliva \\
\hline Positive for $S$. mutans & $39 / 41(95.1 \%)^{\star}$ & $68 / 73(93.2 \%)^{\star}$ & $69 / 78(88.4 \%)^{*}$ \\
\hline Single serotype & $16 / 39(41.0 \%) \dagger$ & $51 / 68(75.0 \%) \dagger$ & $54 / 69(78.3 \%) \dagger$ \\
\hline$c$ & 2 & 43 & 50 \\
\hline$e$ & 14 & 8 & 4 \\
\hline$f$ & 0 & 0 & 0 \\
\hline$k$ & 0 & 0 & 0 \\
\hline Multiple serotype & $21 / 39(53.8 \%) \dagger$ & $17 / 68(25.0 \%) \dagger$ & $15 / 69(21.7 \%) \dagger$ \\
\hline$c$ and $e$ & 14 & 14 & 9 \\
\hline$c$ and $f$ & 0 & 3 & 2 \\
\hline$c$ and $k$ & 2 & 0 & 2 \\
\hline$e$ and $f$ & 0 & 0 & 2 \\
\hline$c, e$ and $f$ & 1 & 0 & 0 \\
\hline$c, e$ and $k$ & 4 & 0 & 0 \\
\hline Serotype undetermined & $2 / 39(5.1 \%) \dagger$ & $0 / 68(0 \%) \dagger$ & $0 / 69(0 \%) \dagger$ \\
\hline
\end{tabular}

*The percentage is expressed in comparison to all $(100 \%)$ of the dental plaque and saliva specimens.

$\dagger$ The percentage is expressed in comparison to all (100\%) of the S. mutans-positive specimens.

the healthy mothers and children $(P<0.05)$. We acknowledge that the control group was not an ideal match for the cardiovascular subjects. However, no difference in $S$. mutans serotype distribution with gender was observed among the children analysed in this study. Also, the serotype distribution patterns of the children and mothers were not significantly different. We surmise, therefore, that more elderly males (matching the cardiovascular test subjects) are also likely to harbour a similar serotype range as the mothers and children employed in this study.
To the best of our knowledge, this is the first report of $S$. mutans serotype distribution in cardiovascular specimens. We previously reported that four blood isolates of $S$. mutans from subjects with bacteraemia after tooth extraction or IE belonged to serotypes $e, f$ or $k$ (Fujiwara et al., 2001; Nakano et al., 2004a). It is generally known that 70 to $80 \%$ of $S$. mutans oral strains belong to serotype c (Hamada et al., 1980; Hirasawa \& Takada, 2003; Shibata et al., 2003; Nakano et al., 2004b), and the saliva samples from the healthy mothers and children were consistent

Table 4. Serotype distribution of $S$. mutans-positive specimens

A significant difference $(P<0.05)$ was found as compared to the oral specimens from the healthy mothers and healthy children by Fisher's exact probability test.

\begin{tabular}{|c|c|c|c|c|c|}
\hline & \multicolumn{2}{|c|}{ Cardiovascular } & \multicolumn{3}{|c|}{ Oral } \\
\hline & $\begin{array}{l}\text { Heart valve } \\
\qquad(n=33)\end{array}$ & $\begin{array}{l}\text { Atheromatous } \\
\text { plaque }(n=32)\end{array}$ & $\begin{array}{c}\text { Cardiovascular surgery } \\
\text { patients }(n=39)\end{array}$ & $\begin{array}{l}\text { Healthy mothers } \\
\qquad(n=68)\end{array}$ & $\begin{array}{l}\text { Healthy children } \\
\qquad(n=69)\end{array}$ \\
\hline$c$ & $10(30.3 \%)^{*} \dagger$ & $21(65.6 \%)^{\star} \dagger$ & $23(59.0 \%)^{\star} \dagger$ & $60(88.2 \%)$ & $63(91.3 \%)$ \\
\hline$e$ & $16(48.5 \%) \dagger$ & $20(62.5 \%)^{\star} \dagger$ & $33(84.6 \%)^{*} \dagger$ & $22(32.4 \%)$ & $15(21.7 \%)$ \\
\hline$f$ & $1(3.0 \%)$ & $2(6.3 \%)$ & $1(2.6 \%)$ & $3(4.4 \%)$ & $4(5.8 \%)$ \\
\hline$k$ & $3(9.1 \%)^{\star}$ & $8(25.0 \%)^{\star} \dagger$ & $4(10.3 \%)^{\star}$ & $0(0 \%)$ & $2(2.9 \%)$ \\
\hline Unserotypable & $8(24.2 \%)^{\star} \dagger$ & $3(9.4 \%)^{\star} \dagger$ & $2(5.1 \%)$ & $0(0 \%)$ & $0(0 \%)$ \\
\hline
\end{tabular}

${ }^{\star}$ Healthy mothers.

$\dagger$ Healthy children. 
with that (Table 3). On the contrary, the detection rate of serotype $c$ in the cardiovascular subjects was significantly lower than that in the healthy subjects (Tables 2 and 3), which led us to speculate that the serotype distribution of $S$. mutans in cardiovascular subjects is significantly different from that in healthy subjects, and that non- $c$ serotypes of $S$. mutans might be possible risk factors for cardiovascular diseases. Further, the number of the subjects with multiple serotypes was higher in the cardiovascular subjects than the healthy subjects, in whom a single serotype $c$ was predominant. In addition, most of the S. mutans-positive specimens showed a positive reaction for at least one of the serotype-specific sets of primers; however, eight (24.2\%) heart valve specimens, three $(9.4 \%)$ atheromatous plaque specimens and two $(5.1 \%)$ dental plaque specimens did not react to any of the serotype-specific sets of primers (Tables 2 and 3). All of these specimens were also confirmed to have a positive reaction to the additional $S$. mutans-specific sets of primers designed based on the nucleotide alignment of the other gene. Also, the nucleotide alignment of those amplicons was shown to be the targeted sequence. Since the sensitivity of all of the PCR methods used in this study was shown to be at nearly the same level (Fig. 1) and the broad-range PCR method also detected the $16 \mathrm{~S}$ rRNA alignment of $S$. mutans, we concluded that at least one additional unknown serotype was present in the cardiovascular specimens.

Serotype $k S$. mutans was first isolated from the blood of IE subjects and characterized by lack of the glucose side chain of the serotype-specific RGPs (Fujiwara et al., 2001), which resulted in a low antigenicity and a low susceptibility to phagocytosis by human polymorphonuclear leukocytes (Nakano et al., 2004b). Serotype $k$ S. mutans strains have also been detected in the oral cavities of healthy children with a prevalence of $2 \%$ (Nakano et al., 2004b). In the present study, there were several $S$. mutans-positive cardiovascular specimens that could not be classified into any of the known serotypes by PCR. We recently analysed the serotypes of 100 S. mutans oral isolates from 100 children, as well as 2500 from 50 children, and all of the strains belonged to the $c, e, f$ or $k$ serotypes (Nakano et al., 2004a), which indicated that the prevalence of a new serotype in the oral cavity would be extremely low, even though it likely exists. If a new serotype of $S$. mutans with a variation of RGPs is found to be selectively present in cardiovascular subjects, then the variation of serotype-specific RGPs may be related to the pathogenesis of $S$. mutans with cardiovascular diseases.

The reason why non-c serotype $S$. mutans was detected more frequently in the cardiovascular specimens than in those from the oral cavity remains to be elucidated. Since serotype-specific RGPs have been shown to be involved with virulence in blood (Tsuda et al., 2000; Engels-Deutsch et al., 2003; Chia et al., 2004; Nakano et al., 2004a), it is possible that an alteration in chemical composition of the RGPs in each serotype is directly related to virulence. Another possible hypothesis is that non- $c$ serotype strains possess common properties other than serotype-specific RGPs, which contribute to their virulence in blood. We previously demonstrated that inactivation of the pac gene encoding the major surface antigen protein antigen $\mathrm{c}$ (PAc) of $S$. mutans resulted in lowered susceptibility to phagocytosis by human polymorphonuclear leukocytes in vitro and a longer duration of bacteraemia in a rat model (Nakano et al., 2006b). In that study, analysis of clinical oral isolates of $S$. mutans without PAc expression showed them to be predominantly detected among the non- $c$ serotypes. In addition, genes encoding collagen-binding adhesins of $S$. mutans were frequently identified in non- $c$ serotype strains (Sato et al., 2004). Although the clinical significance of $S$. mutans detection in cardiovascular specimens remains to be elucidated, subjects with non- $c$ serotype may be at risk for cardiovascular diseases.

\section{ACKNOWLEDGEMENTS}

This study was supported by the 21st Century COE programme entitled 'Origination of Frontier BioDentistry' at Osaka University Graduate School of Dentistry, supported by the Ministry of Education, Culture, Sports, Science and Technology of Japan, as well as a Grant-in-Aid for Scientific Research (B) 16390605 from the Japan Society for Promotion of Science, a Grant-in-Aid for Exploratory Research 17659647 and a Grant-in-Aid for Young Scientists (A) 18689050 from Ministry of Education, Culture, Sports, Science and Technology of Japan.

\section{REFERENCES}

Chia, J. S., Lin, Y. L., Lien, H. T. \& Chen, J. Y. (2004). Platelet aggregation induced by serotype polysaccharides from Streptococcus mutans. Infect Immun 72, 2605-2617.

Engels-Deutsch, M., Pini, A., Yamashita, Y., Shibata, Y., Haikel, Y., Scholler-Guinard, M. \& Klein, J. P. (2003). Insertional inactivation of pac and $r m l B$ genes reduces the release of tumor necrosis factor alpha, interleukin-6, and interleukin-8 induced by Streptococcus mutans in monocytic, dental pulp, and periodontal ligament cells. Infect Immun 71, 5169-5177.

Fujiwara, T., Nakano, K., Kawaguchi, M., Ooshima, T., Sobue, S., Kawabata, S., Nakagawa, I. \& Hamada, S. (2001). Biochemical and genetic characterization of serologically untypable Streptococcus mutans strains isolated from patients with bacteremia. Eur J Oral Sci 109, 330-334.

Gauduchon, V., Benito, Y., Celard, M., Mouren, C., Delorme, V., Phillippe-Bert, J., Etienne, J. \& Vandenesch, F. (2001). Molecular diagnosis of recurrent Streptococcus mutans endocarditis by PCR amplification and sequencing. Clin Microbiol Infect 7, 36-37.

Hamada, S. \& Slade, H. D. (1980). Biology, immunology, and cariogenicity of Streptococcus mutans. Microbiol Rev 44, 331-384.

Hamada, S., Masuda, N. \& Kotani, S. (1980). Isolation and serotyping of Streptococcus mutans from teeth and feces of children. J Clin Microbiol 11, 314-318.

Hirasawa, M. \& Takada, K. (2003). A new selective medium for Streptococcus mutans and the distribution of S. mutans and S. sobrinus and their serotypes in dental plaque. Caries Res 37, 212-217.

Hoshino, T., Kawaguchi, M., Shimizu, N., Hoshino, N., Ooshima, T. \& Fujiwara, T. (2004). PCR detection and identification of oral 
streptococci in saliva samples using gtf genes. Diagn Microbiol Infect Dis 48, 195-199.

Nakano, K., Nomura, R., Nakagawa, I., Hamada, S. \& Ooshima, T. (2004a). Demonstration of Streptococcus mutans with a cell wall polysaccharide specific to a new serotype, $k$, in the human oral cavity. J Clin Microbiol 42, 198-202.

Nakano, K., Nomura, R., Shimizu, N., Nakagawa, I., Hamada, S. \& Ooshima, T. (2004b). Development of a PCR method for rapid identification of new Streptococcus mutans serotype $k$ strains. J Clin Microbiol 42, 4925-4930.

Nakano, K., Inaba, H., Nomura, R., Nemoto, H., Takeda, M., Yoshioka, H., Matsue, H., Takahashi, T., Taniguchi, K. \& other authors (2006a). Detection of cariogenic Streptococcus mutans in extirpated heart valve and atheromatous plaque specimens. J Clin Microbiol 44, 3313-3317.

Nakano, K., Tsuji, M., Nishimura, K., Nomura, R. \& Ooshima, T. (2006b). Contribution of cell surface protein antigen PAc of Streptococcus mutans to bacteremia. Microbes Infect 8, 114-121.

Nomura, R., Nakano, K., Nemoto, H., Fujita, K., Inagaki, S., Takahashi, T., Taniguchi, K., Takeda, M., Yoshioka, H. \& other authors (2006). Isolation and characterization of Streptococcus mutans in heart valve and dental plaque specimens from a patient with infective endocarditis. J Med Microbiol 55, 1135-1140.

Oho, T., Yamashita, Y., Shimazaki, Y., Kushiyama, M. \& Koga, T. (2000). Simple and rapid detection of Streptococcus mutans and
Streptococus sobrinus in human saliva by polymerase chain reaction. Oral Microbiol Immunol 15, 258-262.

Rovery, C., Greub, G., Lepidi, H., Casalta, J. P., Habib, G., Collart, F. \& Raoult, D. (2005). PCR detection of bacteria on cardiac valves of patients with treated bacterial endocarditis. J Clin Microbiol 43, 163-167.

Sato, Y., Okamaoto, K., Kagami, A., Yamamoto, Y., Igarashi, T. \& Kizaki, H. (2004). Streptococcus mutans strains harboring collagenbinding adhesin. J Dent Res 83, 534-539.

Scannapieco, F. A., Bush, R. B. \& Paju, S. (2003). Association between periodontal disease and risk for atherosclesosis, cardiovascular disease, and stroke. A systematic review. Ann Periodontol 8, 38-53.

Shibata, Y., Ozaki, K., Seki, M., Kawato, T., Tanaka, H., Nakano, Y. \& Yamashita, Y. (2003). Analysis of loci required for determination of serotype antigenicity in Streptococcus mutans and its clinical utilization. J Clin Microbiol 41, 4107-4112.

Tsuda, H., Yamashita, Y., Toyoshima, K., Yamaguchi, N., Oho, T., Nakano, Y., Nagata, K. \& Koga, T. (2000). Role of serotype-specific polysaccharide in the resistance of Streptococcus mutans to phagocytosis by human polymorphonuclear leukocytes. Infect Immun 68, 644-650.

Ullman, R. F., Miller, S. J., Strampfer, M. J. \& Cunha, B. A. (1988). Streptococcus mutans endocarditis: report of three cases and review of the literature. Heart Lung 17, 209-212.

Vose, J. M., Smith, P. W., Henry, M. \& Colan, D. (1987). Recurrent Streptococcus mutans endocarditis. Am J Med 82, 630-632. 